Página inicial: 35 - Página final: 51 TIPO DE ARTíCULO: de Investigación

\title{
Prácticas educativas familiares y desempeño académico en adolescentes escolarizados en el grado noveno de instituciones educativas oficiales del municipio de Envigado.
}

\author{
Family Educational Practices and Academic \\ Performance in Schooled Adolescents in the Ninth Grade in Public \\ Educational Institutions in the Municipality of Envigado.
}

Recibido: Enero 2008

revisado: Junio 2008 aceptado: Enero 2009
Por: Luz Magnolia Tilano' Gloria Cecilia. Henao ${ }^{2}$ Julián A. Restrepo ${ }^{3}$

\section{Resumen:}

En el presente articulo, se plantea la relación entre las prácticas educativas familiares y el desempeño académico de 451 adolescentes ( hombres y mujeres ) de noveno grado de Instituciones oficiales del Municipio de Envigado/Antioquia. Con una selección aleatoria simple y la aplicación de las escalas de afecto (EA-P) y de normas y exigencias (ENE-P) de Bersabé, Fuentes y Montrico (2001) con hijos y padres, se obtiene que los estilos de autoridad inductivos favorecen el desempeño académico, mientras que los rígidos propician un desempeño académico deficitario, siendo mas bajo en los permisivos. Además, los padres son percibidos más permisivos y rígidos que las madres, finalmente se observan divergencias entre las percepciones de los hijos y los padres, quienes tienden a evaluarse de manera positiva.

\section{Palabras Claves:}

Prácticas Educativas Familiares, Desempeño Académico, Afecto, Normas y exigencias.

\section{Abstract:}

In this article, the authors pose the relationship between the family educational practices and the academic performance of 451 adolescents (men and women) in the ninth grade of public institutions of the Municipality of Envigado, Antioquia. With an at random simple selection and the application of the Parental Affection Scale and the Parental Regulation and Demand Scales by Bersabé, Fuentes and Montrico (2001) with children and parents, it is obtained that the styles of inductive authority favor the academic performance, while the rigid styles create a poor academic performance, which is the lowest in permissive parents. Furthermore, it is male parents who are perceived as being permissive and demanding than female parents. Lastly, it can be observed some divergences between the children's and the parents' perceptions, the latter tend to assess themselves positively.

\section{Key words:}

Family educational practices, Academic performance, Affection, regulations and demands.

\footnotetext{
${ }^{1}$ Magíster en Psicología. Docente Universidad de San Buenaventura. luz.tilano@usbmed.edu.co

${ }^{2}$ PHD en ciencias sociales. Magíster en neuropsicología. Educación especial.gloria.henao@usbmed.edu.co

${ }^{3}$ Estudiante de décimo semestre de Psicología. Joven Investigador
} 


\section{Introducción}

El Desempeño Académico manifiesta en forma estimativa lo que una persona ha aprendido como consecuencia de un proceso de instrucción y formación (Pizarro1985, citado por Andrade), en los centros oficiales de enseñanza normalmente se expresa a través de las calificaciones (Martínez 1996) y está determinado por factores como el status socioeconómico familiar, el nivel educativo de los padres, las estrategias de enseñanza-aprendizaje, la relación docente-dicente, la motivación del educando, los estilos parentales, las pautas de crianza, el exceso de estudiantes por aula, la cantidad de materias que deben ver los alumnos, entre otros.

Entre estos, la familia entendida como un grupo o sistema compuesto por subsistemas que serían sus miembros y a la vez integrada a un sistema que es la sociedad (Ares, citado por Herrera, 1997) se constituye como elemento esencial para entender el fenómeno del rendimiento académico, pues cada miembro de este sistema posee roles que varían en el tiempo y que dependen de la edad, el sexo y la interacción con los otros miembros familiares (Seguel, Holmes, Venegas, Alcérreca, y Salamanca, 2000), por tanto, las influencias dentro de la familia no son unidireccionales sino que son una red donde todos sus integrantes influyen sobre los otros, es así como todos los sucesos que se encuentran inmersos en el sistema familiar inciden en los procesos por los cuales atraviesan los subsistemas del mismo, en este caso, los hijos durante la vida escolar.

Desde este marco global, teniendo en cuenta el desempeño académico como un constructo complejo que viene determinado por la interacción de variables como la inteligencia, la motivación, la personalidad, las actitudes, los contextos entre otros, (Adell, 2002), y además a la familia como elemento dinamizador del proceso educativo, surge el interés de identificar la relación existente entre las prácticas educativas familiares y el desempeño académico de los hijos adolescentes que se encuentran escolarizados.

Lamborn, Darling, Steimberg \& Dombush (1991) con una muestra de 6400 adolescentes de nueve colegios de Wisconsin y California, encontraron que aquellos estudiantes que son educados en hogares autoritativos consiguen mejores logros académicos, un mejor desarrollo social y una adecuada autoestima que los sometidos a estilos autoritarios que muestran menor ajuste social, el mÁs bajo rendimiento se vio en aquellos expuestos a estilos permisivos o no implicados, los cuales muestran menos persistencia en sus tareas. En este mismo orden Xinyin Chen (1997) y Londoño (2005), estudiando niveles académicos básicos, encontraron que la presencia de los padres juega un papel importante en la educación y que el rendimiento

académico se encuentra afectado por aquellas prácticas parentales donde los padres no se involucran en el proceso educativo. Además que los padres que participan activamente en la 
realización de tareas escolares con sus hijos y utilizan en la práctica disciplinaria métodos inductivos, de refuerzos frente a las conductas adecuadas y poco castigo físico actúan como favorecedores del proceso de aprendizaje.

El afecto y el clima familiar han sido también relacionados con el rendimiento académico; Casanova, García, De la Torre \& De la Villa (2005) notaron que en alumnos con logros académicos normales, las variables socio-demográficas tienen un mayor poder de predicción con respecto al rendimiento académico, mientras que en estudiantes con logros académicos deficitarios las variables de tipo familiar jugaban un rol más importante. De esta manera, factores sociodemográficos como el nivel escolar de los padres, el status socioeconómico familiar y factores personales como la motivación, el género y las relaciones sociales en clase (Lozano, (2003), Steimberg, Dornbush \& Brown, 1992 ) juegan un papel esencial en dicho proceso y permiten identificar dos categorías básicas, la primera referente a características estructurales de la familia y la segunda relacionada con un lado más dinámico de ésta, como lo es el clima familiar (Covadonga, 2001).

La violencia intrafamiliar y más específicamente el maltrato físico y emocional, infligido por los padres, madres y tutores, y de igual manera la frecuencia en que se presenta este maltrato, se encuentra asociada también al bajo desempeño académico de los menores escolarizados, Espinoza (2006) tras estudiar a 400 estudiantes de primer grado de secundaria de establecimientos educativos de Guatemala, resalta la violencia como un elemento simultáneo a la práctica de un modelo autoritario de enseñanza aprendizaje y su respectiva relación con el bajo desempeño académico.

Por otra parte, algunas investigaciones señalan la existencia de diferencias y convergencias entre padres y madres, con relación al estilo en que manifiestan dichas prácticas educativas, Gracia, Lila \& Musitu (2005) en la investigación "Rechazo parental y ajuste psicológico y social de los hijos" encontraron que las características de personalidad de los niños rechazados difieren significativamente de las de los niños cuyas relaciones con sus padres no presentan características disfuncionales ya que los primeros tienden a reaccionar con manifestaciones hostiles y agresivas, muestran una escasa confianza tanto en otras personas como fuente de seguridad, confianza y apoyo, como en sus sentimientos de estima, aceptación, y competencia, además, no se encontraron diferencias significativas en las dimensiones de aceptación/rechazo materno ni paterno entre niños y niñas. En contraste, en algunas investigaciones anteriores a esta, se determinó que las percepciones de padres e hijos con relación a la conducta del padre y de la madre difieren significativamente en la dimensión calor/afecto, pero que no aparecen diferencias en la dimensión rechazo y que los niños perciben un mayor calor y afecto por parte de sus madres. De igual forma, hallaron diferencias significativas entre la percepción de los hijos del trato que reciben de la madre y la evaluación 
de los padres, donde la evaluación de los padres es más positiva, en el sentido de un mayor calor y afecto y un menor rechazo hacia los hijos (Gracia, 2002) y además la comunicación se evidencia más elevada en dirección a la madre que con el padre (Pérez, Fuster \& Musitu, 1988) Estas investigaciones evidencian la necesidad de profundizar aspectos relacionados con el estilo parental, como son características de comunicación y afecto intrafamiliar en relación con el rendimiento académico.

\section{Practicas Educativas Familiares}

Las practicas educativas se entienden como todos aquellos procesos formativos que pueden presentarse en diversos contextos del desarrollo humano (cultural, social, escolar, familiar), que permiten la adquisición de diferentes formas de educación (Quintana, 1993) y promueven el desarrollo personal de los individuos teniendo en cuenta el aprendizaje que se realiza como una construcción y una apropiación personal que lo lleva a efectuar una reestructuración del conocimiento del que dispone (Coll, Miras, Onrubia \& Solé, 1998).

Las prácticas educativas familiares hacen referencia a los esquemas prácticos de conducta que reducen las múltiples pautas educativas paternas a unas pocas dimensiones básicas que cruzadas entre sí, dan lugar a diversos tipos habituales de educación familiar (Quintana, 1993), dentro de los cuales se identifican no sólo la percepción y aplicación de la norma dentro del hogar, sino también otras variables implicadas en la relación padre e hijo como el nivel afectivo y comunicativo y el grado de aceptación tanto de los padres hacia los hijos como de estos últimos hacia sus padres.

Siguiendo a Moreno y Cubero (1990) se identifica el afecto como una dimensión en la relación parental que actúa como un elemento dinamizador de los diferentes procesos que se encuentran inmersos en el sistema familiar, puede ser entendido como todas aquellas conductas que los padres manifiestan a sus hijos a través de las cuales estos últimos perciben que son queridos y aceptados como integrantes del núcleo familiar (Sorribes \& García, 1996). Se divide en dos subdimensiones: La relación bipolar existente entre aceptación-rechazo que se manifiesta a partir de conductas de inclusión de los hijos en la dinámica familiar, el cariño, la expresión de sentimientos o a través de expresiones cargadas de contenido hostil, agresividad, indiferencia y exclusión con respecto a la participación del hijo en situaciones familiares como la toma de decisiones, la negociación de la norma etc. y la relación entre sobreprotección-distanciamiento donde se pone en juego un estilo parental aglutinador frente a otro totalmente desligado y desinteresado.

Otra dimensión importante es el grado de control que los padres utilizan para educar a sus hijos, es decir, el nivel en que se manifiesta el proceso normativo y formativo por parte de los padres 
(Sorribes \& García, 1996). De este modo, el uso de una adecuada combinación de firmeza y razonamiento por parte de los padres, permite que sus hijos adquieran un autocontrol adecuado más que cuando se interviene desde estilos más de corte autoritario y permisivo (Moreno y Cubero, 1996).

Para Moreno y Cubero (1990) la capacidad de establecer un ambiente comunicativo es otra de las dimensiones que ejerce una significativa influencia dentro de la dinámica familiar y por ende en las prácticas educativas que se desarrollen en este contexto. La comunicación debe concebirse en el ámbito familiar como la posibilidad de crear un ambiente dinámico que permita generar un proceso de implicación donde tanto los hijos como los padres participen en el abordaje de la norma de una manera explicita y racional, donde se respeta y se tiene en cuenta el punto de vista del otro y donde ambas partes influyen de manera equilibrada en la toma de decisiones. Se trata entonces de una dinámica que permite compartir problemas, conflictos, dudas, ansiedades, expectativas y satisfacciones (Moreno y Cubero, 1996), vivencias que son propias de ciclos determinantes del desarrollo como la niñez y la adolescencia y que requieren de un adecuado abordaje y orientación por parte de los padres.

\section{Disciplina Familiar: Normas Y Exigencias}

En la actualidad es posible identificar diversas clasificaciones referidas a los estilos que los padres asumen dentro de su rol formativo, así pues, se encuentran denominaciones como las utilizadas por Bersabé, Fuentes y Montrico (2001) en las Escalas de Afecto (EA) y la Escala de Normas y Exigencias (ENE) en las cuales se refieren a los estilos rígido, inductivo, indulgente que suponen diferentes características tanto en los padres como en los hijos dentro de las practicas educativas familiares y buscan establecer de una manera más específica todos aquellos comportamientos que ambas partes manifiestan dentro del proceso educativo en el contexto familiar. La clasificación más referenciada en los estudios es la de Baumrind (1973) que identificó tres tipos de control parental que difirieren a nivel cualitativo el uno del otro: democrático, autoritativo y permisivo. Este modelo conjuga la identificación afectiva y el control comportamental, así, por un lado debe identificarse el elemento afectivo entre padres e hijos y por otro debe existir cierto nivel de control adulto, compuesto por diversas técnicas y estilos, que permita la regulación de la norma dentro del contexto familiar y finalmente en los procesos inmersos dentro de los demás contextos.

\section{Practicas Educativas Familiares Y Desempeño Académico}

El ambiente se define como una disposición de estructuras seriadas e interdependientes cada una de las cuales se incluye en la siguiente y en las que puede o no participar la persona en desarrollo, es decir, una estructura múltiple de entornos en interacción, en cada uno de los 
cuales participan a su vez múltiples personas igualmente en interacción que ejercen una importante influencia en el individuo (Clemente \& Hernández, 1996).

A estas estructuras seriadas e interdependientes, Bronfenbrenner (1979) las ha denominado sistemas ambientales, microsistema, mesosistema, exosistema y macrosistema.

El microsistema constituye uno de los niveles más internos del ambiente y se define como "un patrón de actividades, roles y relaciones interpersonales que la persona en desarrollo experimenta en un entorno determinado, con características físicas y materiales particulares" (Bronfenbrenner, 1979). Algunos contextos pertenecientes al microsistema son la familia del alumno, sus pares, la escuela y el vecindario, dentro del los cuales el individuo tiene interacciones directas con sus padres, pares, maestros y otros cercanos.

El mesosistema constituye el segundo nivel ecológico propuesto por Bronfenbrenner (1979) quien lo describe como aquel ambiente que "comprende las interrelaciones de dos o más entornos en los que la persona participa activamente (por ejemplo, para un niño, las relaciones entre el hogar, la escuela y el grupo de pares del barrio; para un adulto, entre la familia, el trabajo y la vida social)". Desde este marco contextual, el mesositema es el foco central de la presente investigación ya que no solo involucra los vínculos si no también las relaciones existentes entre los microsistemas, es decir, acoge la posibilidad de que la experiencia vivida por el individuo en un microsistema influya en la experiencia de otro, para el caso, el mesosistema permite dar una explicación a la relación existente entre el rendimiento escolar y las practicas educativas familiares.

De lo anterior se infiere que el potencial evolutivo de un mesositema permite que se genere una vinculación positiva entre los microsistemas de los que se compone, así pues, se hablaría de una relación directamente proporcional entre el grado de aprecio, de comunicación, de conocimiento mutuo y de realización de actividades conjuntas entre las personas con el individuo en desarrollo y la capacidad de estos entornos para promover dicho desarrollo (Hernández, 1996).

Bronfenbrenner (1979) afirma que un alto grado de vinculación y armonía entre los entornos genera un impacto positivo más significativo en aquellos individuos en desarrollo que poseen bajos niveles de competencia, que en aquellos con un alto nivel de competencia. Por ejemplo aquellos alumnos que mantienen una relación con sus padres caracterizada por el rechazo, posiblemente tengan gran dificultad para relacionarse con sus compañeros o docentes y viceversa.

AGO.USB Medellin-Colombia V. $9 \quad N^{\circ} 1$ PP. 1-294 Enero-Junio 2009 ISSN: 1657-8031 
El exosistema esta constituido por los contextos que "no incluyen a la persona en desarrollo como participante activo, pero en los cuales se cruzan hechos que afectan a lo que ocurre en el entorno y que comprende a la persona en desarrollo, o que se ven afectados por lo que ocurre en ese entorno" (Bronfenbrenner, 1979), en ese sentido, algunos de los posibles exosistemas para un sujeto en edad escolar podrían ser el lugar de trabajo de los padres, los amigos de la familia, o en el contexto educativo, los diversos comités conformados en una institución educativa.

El macrosistema se refiere a todos aquellos aspectos culturales y etnográficos de los cuales el individuo recibe una influencia significativa, que acoge tanto los valores como las costumbres y que difieren entre lugares. Constituye un entorno global que permite entender la homogeneidad entre los demás subsistemas (Hernández, 1996), es decir, la identificación de una notable influencia de aspectos como las creencias, actitudes, tradiciones, leyes etc. de una cultura en otros sistemas menores como el colegio, la familia y el individuo propiamente.

\section{Metodologia}

\subsection{Diseño Metodológico}

En un estudio empírico analítico de tipo observacional correlacional y de corte trasversal se pretende identificar la relación existente entre las prácticas educativas familiares y el desempeño académico en adolescentes de las instituciones educativas oficiales del Municipio de Envigado.

\subsection{Población y Muestra}

La totalidad de la población estudiada se conforma por los adolescentes matriculados en el grado noveno en las instituciones educativas oficiales del Municipio de Envigado/Antioquia durante el año 2006.

La muestra está constituida por 451 alumnos adolescentes y sus respectivos padres los cuales pertenecen a diversos estratos socioeconómicos. La elección fue aleatoria simple, entre los diversos cursos del grado noveno de cada institución educativa. 


\subsection{Variables}

\begin{tabular}{|l|l|}
\hline VARIABLE & CONCEPTO \\
\hline PERIODO DEL & $\begin{array}{l}\text { Definida según los años cumplidos por el sujeto al } \\
\text { momento de ser evaluado (Adolescencia). }\end{array}$ \\
\hline $\begin{array}{l}\text { ESCOLARIDAD } \\
\text { ALCANZADA }\end{array}$ & Último grado de educación básica en curso (Noveno) \\
\hline RENDIMIENTO & $\begin{array}{l}\text { El producto que rinde o da el alumnado en el ámbito de los } \\
\text { centros oficiales de enseñanza y que normalmente se } \\
\text { expresa a través de las calificaciones escolares (Martínez, } \\
1996)\end{array}$ \\
\hline PRÁCTICAS & $\begin{array}{l}\text { Los esquemas prácticos de conducta que reducen las } \\
\text { múltiples pautas educativas paternas a unas pocas } \\
\text { dimensiones básicas que, cruzadas entre sí, dan lugar a } \\
\text { diversos tipos habituales de educación familiar (Quintana, } \\
\text { 1993), dentro de los cuales se identifican no sólo la } \\
\text { percepción y aplicación de la norma dentro del hogar si no } \\
\text { también otras variables implicadas en la relación padre e } \\
\text { hijo como el nivel afectivo y comunicativo y el grado de } \\
\text { aceptación tanto de los padres hacia los hijos como de } \\
\text { estos últimos hacia sus padres. }\end{array}$ \\
\hline
\end{tabular}

\subsection{Instrumentos}

\subsubsection{ESCALA DE NORMAS Y EXIGENCIAS PARA HIJOS (ENE-H) (Bersabé, Fuentes y Montrico, 2001):}

Consiste en una escala tipo Likert, que presenta cinco opciones de respuesta (Nunca, Pocas veces, Algunas veces, a menudo, Siempre) y se encuentra compuesta por 28 ítems. El número de factores corresponden a los prototipos de autoridad parental descritos por Baumrind (1971). Da lugar a tres factores referentes a la forma de imponer y exigir las normas:

Factor I (forma inductiva): Los padres explican a sus hijos el establecimiento de las normas, y las adaptan a las necesidades y posibilidades de sus hijos. 
Factor II (forma rígida): Los padres imponen a sus hijos el cumplimiento de las normas, y mantienen un nivel de exigencias demasiado alto e inadecuado a las necesidades de los hijos. Factor III (forma indulgente): Los padres no ponen normas ni límites a la conducta de sus hijos y, si lo hacen, no exigen su cumplimiento.

En cada factor, la fiabilidad como consistencia interna es bastante elevada (Factor I-padre a $=0,85$; Factor II-padre $a=0,73$; Factor III-padre $a=0,60$; Factor I-madre $a=0,80$; Factor IImadre $a=0,72$; Factor III-madre $a=0,64$ ).

\subsubsection{ESCALA DE AFECTO PARA HIJOS (EA-H) (Bersabé, Fuentes y Montrico, 2001):}

Consiste en una escala tipo Likert, que presenta cinco opciones de respuesta (Nunca, Pocas veces, Algunas veces, A menudo, Siempre) y se encuentra compuesta por 20 ítems. Esta formada por:

Factor I (afecto-comunicación): Afecto, interés y comunicación que manifiestan los padres a sus hijos.

Factor II (crítica-rechazo): Crítica, rechazo y falta de confianza de los padres hacia sus hijos.

En cada factor, la fiabilidad como consistencia interna es bastante elevada (Factor I-padre a $=0,90$; Factor II-padre a =0,83; Factor I-madre a =0,87; Factor II-madre $a=0,81$ ).

\subsubsection{ESCALA DE AFECTO PARA PADRES (EA-P) Y ESCALA DE NORMAS $Y$ EXIGENCIAS PARA PADRES (ENE-P) (Bersabé, Fuentes y Montrico, 2001):}

Es una versión que contestan los padres, se componen de los mismos ítems de la diseñada para el hijo. Las puntuaciones y las distribución de las preguntas para cada factor en estas escalas es igual que en las de los hijos. Los coeficientes de fiabilidad de la versión para padres tienen una adecuada consistencia interna ( $a=0,78$ en afecto-comunicación; $a=0,66$ en crítica-rechazo; $a$ $=0,68$ en forma inductiva; $a=0,68$ en forma rígida; y $a=0,60$ en forma indulgente) aunque algo menor que en la versión para hijos. 


\subsubsection{Informe de calificaciones escolares}

Los informes de calificaciones esclares fueron proveídos por cada una de las instifuciones educativas, en los cuales se encuentran asignados las calificaciones a nivel cuantitativo por materia y su respectivo promedio.

\section{Resultados}

En la Tabla 1 se presentan las medias, las desviaciones estándar y el coeficiente de relación ANOVA de todas las variables de estudio, Se observa que aquellos estudiantes con desempeño académico alto tienen menor percepción de crítica rechazo por parte de su padre $(16,90)$ y su madre $(16,90)$ a diferencia de aquellos estudiantes con niveles académicos medios que perciben esta variable en niveles altos, tanto en el padre $(18,71)$ como en la madre $(19,22)$ y aun más altos en aquellos alumnos con un desempeño académico bajo (Padre=20,03 Madre=9,81). De aquí se rescata también que la percepción de crítica/rechazo por parte de los hijos, es mayor en el padre que en la madre.




Con respecto a la percepción que el hijo tiene de la comunicación y el afecto en el hogar se observa que los aquellos estudiantes con un desempeño académico bajo evidencian niveles afectivos y comunicativos más bajos (Padre $=32,67$ - Madre $=38,14$ ) y que los estudiantes con un desempeño medio perciben un clima familiar más comunicativo y afectivo (Padre=34,83 Madre $=40,01$ ) pero no mayor que en aquellos estudiantes con desempeño académico alto (Padre $=35,90-$ Madre $=41,48$ ), de esta variable se destaca también que hijos perciben niveles más altos de afecto y comunicación en la relación con su madre, en comparación con su padre y que las puntuaciones en esta variable tienen un aumento directamente proporcional con el nivel de desempeño académico.

Por otra parte, con relación a los estilos de autoridad, se encuentra que los hijos que tiene un nivel de desempeño académico alto perciben mayores niveles de Forma Inductiva en el estilo de autoridad de sus padres, pero que este nivel varía entre el padre $(38,57)$ y la madre $(41,40)$, y es percibido en un nivel menor en aquellos estudiantes que tienen un desempeño académico bajo (Padre=35,70 - Madre=38,17) pero menos bajo en aquellos estudiantes con niveles académicos medios (Padre=38,90-Madre=40,41). De igual manera es relevante identificar que la forma inductiva tiene más presencia en la madre que en el padre.

De los otros estilos de autoridad se encuentra relevante que en aquellas familias donde se utiliza un estilo rígido los resultados académicos de los hijos son mayores en comparación con aquellas en las que predomina un estilo indulgente y que en estas variables los padres obtuvieron una puntuación más alta que las madres, es decir, los padres son percibidos más rígidos y permisivos que las madres.

\begin{tabular}{|c|c|c|c|c|c|c|c|c|c|c|c|c|}
\hline Medias, & Pesvi & acion & es Están & dar y & $\begin{array}{l}\text { bla } 2 \\
\text { Sign }\end{array}$ & ificancia & de lo & 5 var & iables & NOV & & \\
\hline PRACTICAS & & & & REN & IMIE & NTO AC & ADEM & co & & & & \\
\hline VARIABLE & $\mathbf{N}$ & & BAJO & & & MEDIO & & & ALTO & & $f$ & $p$ \\
\hline & & $\mathbf{N}$ & Media & DS & $\mathbf{N}$ & Media & DS & $\mathbf{N}$ & Media & DS & & \\
\hline $\begin{array}{l}\text { Utilización del afecto y } \\
\text { la comunicación en la } \\
\text { relación }\end{array}$ & 405 & 133 & 42,47 & 6,80 & 130 & 42,88 & 5,70 & 142 & 42,48 & 7,43 & 0,16 & 0,85 \\
\hline $\begin{array}{l}\text { Utilización de crítica y } \\
\text { rechazo en la relación }\end{array}$ & 405 & 133 & 18,34 & 5,43 & 130 & 17,58 & 4,06 & 142 & 17,46 & 6,63 & 1,18 & 0,31 \\
\hline $\begin{array}{l}\text { Estilo en el } \\
\text { establecimiento de las } \\
\text { normas y exigencias } \\
\text { (Forma INDUCTIVA) }\end{array}$ & 400 & 131 & 44,05 & 5,82 & 129 & 43,68 & 5,36 & 140 & 43,68 & 5,36 & 0,15 & 0,86 \\
\hline $\begin{array}{l}\text { Estilo en el } \\
\text { establecimiento de las } \\
\text { normas y exigencias } \\
\text { (Forma RIGIDA) }\end{array}$ & 400 & 131 & 26,78 & 6,04 & 129 & 27,52 & 6,20 & 140 & 26,22 & 6,66 & 1,42 & 0,24 \\
\hline $\begin{array}{l}\text { Estilo en el } \\
\text { establecimiento de las } \\
\text { normas y exigencias } \\
\text { (Forma INDULGENTE) }\end{array}$ & 399 & 130 & 17,28 & 3,81 & 129 & 17,22 & 4,46 & 140 & 16,21 & 3,83 & 3,02 & 0,05 \\
\hline
\end{tabular}


En la tabla 2 se muestra la percepción de los padres con respecto a las prácticas parentales estudiadas y se identifica que los padres perciben menores niveles de crítica / rechazo y formas rígida e indulgente en sus prácticas parentales y mayores niveles de afecto y comunicación al igual que el manejo de un estilo inductivo.

Se puede apreciar que los valores en cada una de las variables evaluadas por los padres presentan gran semejanza y que las diferencias entre cada una no son realmente significativas.

6. Discusión y conclusiones:

El presente estudio muestra cómo aspectos propios de las prácticas educativas familiares se relacionan con el desempeño académico de los adolescentes escolarizados en el municipio de Envigado/Antioquia y articula sus resultados con otros estudios realizados en Colombia y el mundo, a la vez que los analiza a la luz de algunos postulados teóricos.

Los datos obtenidos apoyan los postulados teóricos de Baurimd (1973), retomados por Londoño (2005), quien describe que el bajo desempeño académico de los hijos se encuentra afectado por aquellas prácticas parentales donde los padres no se involucran en el proceso educativo y que aquellos padres que participan activamente en el proceso académico de sus hijos y utilizan en la práctica disciplinaria métodos inductivos actúan como favorecedores del proceso de aprendizaje, lo que permite inferir que el resultado escolar depende en gran medida de los estilos de autoridad ejercidos por estos y que las características de cada uno están implicadas en el proceso de aprendizaje.

En este marco general, el estilo inductivo (Bersabé, Fuentes y Montrico, 2001), denominado por Baumrind (1973) como el estilo democrático, en el cual los padres se esmeran por ser coherentes, afectivos y justos con sus hijos, propicia todos los elementos necesarios para el alcance de los logros escolares de éstos, se observa como entre estas dos variables se manifiesta una relación que indica que entre más sea utilizado el estilo inductivo mayor es la posibilidad de aumentar el desempeño escolar en los adolescentes.

Se encuentra relación entre el estilo de autoridad indulgente (permisivo) con un desempeño menor a nivel académico, a diferencia del rígido (autoritario) cuyo desempeño académico es mayor, lo cual se plantea en estudios como el realizado por Lamborn, Darling, Steimberg \& Dombush (1991) donde se identifica que los estudiantes educados bajo estilos parentales autoritarios son menos ajustados socialmente, pero obtienen un mejor desempeño académico que aquellos educados en hogares permisivos, los cuales muestran menor persistencia a este nivel. 
Desde esta óptica se observa que aquellos adolescentes que carecen de acompañamiento familiar carecen también de acompañamiento académico y a diferencia de aquellos pertenecientes a una estructura rígida donde se presenta un acompañamiento académico con una gran carga de imposición y hostilidad, tienen un desempeño académico más deficitario.

Teniendo en cuenta el postulado teórico de Bronfenbrenner (1979) la familia como uno de los principales microsistemas en los que interactúa el individuo, juega un papel primordial en el desarrollo de interacciones con otros microsistemas como la escuela, y por ende cuando aparece una situación (como puede ser bajo desempeño académico) éste puede ser concebido como un indicador de una disfunción familiar (evidenciada en la prácticas educativas familiares), donde no se debe ver el individuo como el problemático, sino como el portador de las problemáticas familiares (Molina citado por Herrera, 1997).

Teniendo en cuenta el referente de Bronfenbrenner, se retoma el mesosistema como aquella estructura que permite realizar una asociación entre los microsistemas y explicar como la experiencia vivida en microsistema como el familiar influye en la experiencia del microsistema escolar. Así pues, se nota que el potencial evolutivo del mesositema (relación familia-desempeño académico) es decir la conjugación de grado de aprecio, de comunicación, de conocimiento mutuo y de realización de actividades conjuntas entre los integrantes del microsistema familiar (forma inductiva) con el hijo escolarizado promueven un adecuado desarrollo en el microsistema escolar que es reflejado en el desempeño académico.

Referente a los estilos permisivo y rígido, los resultados de la presente investigación apoyan también la teoría de sistemas, en la medida en que se logra evidenciar que aquellos alumnos que mantienen una relación con sus padres caracterizada por el rechazo, generalmente tienden a poseer niveles bajos de competencia en otros microsistemas como el escolar, no solo a nivel académico si no también en el área social.

Al igual que en el estudio realizado por Gracia (2002) se identifican algunas diferencias significativas entre la percepción de los hijos y la de los padres con respecto a variables como afecto y crítica/rechazo, ya que la evaluación de los padres es más positiva, con respecto al afecto y la aceptación y menor en el rechazo, lo cual evidencia en los padres una propensión a evaluarse de forma más positiva que los hijos (más afectuosos y menos rechazantes), según Gracia (2002) influenciado por un componente de deseabilidad manifiesto tanto en el padre como en la madre.

En congruencia con el estudio mencionado anteriormente, se encuentra que los hijos perciben diferencias en su relación con el padre y la madre que se refieren básicamente a una percepción 
de afecto más acentuada en dirección a la madre que al padre, al igual que niveles de comunicación más elevados en la primera, aunque es importante resaltar que existe una discrepancia en la variable crítica/rechazo, ya que mientras en el estudio realizado por Gracia (2002) no se evidencia ninguna diferencia, en el presente estudio se observaron mayores niveles de crítica y rechazo en el padre que en la madre.

Finalmente, se identifica que altos niveles de afecto y comunicación son percibidos de manera más acentuada en aquellos estudiantes que alcanzaron un alto desempeño académico, debido a que estas variables operan como factores dinamizadores de la relación paterno-filial, implican a los hijos en la dinámica familiar (Moreno \& Cubero, 1990) e influyen directamente en los procesos que el estudiante lleva a cabo en el microsistema escolar (Bromfenbrenner, 1979), además, que la utilización de una adecuada combinación de firmeza y razonamiento por parte estos padres inductivos, permite que sus hijos adquieran un autocontrol y desempeño adecuados más que cuando se interviene desde estilos más de corte autoritario y permisivo (Moreno y Cubero, 1996). 


\section{Referencias Bibliográficas}

Hernández, G. E. (1998). Una aproximación al análisis cualitativo de los datos. En: Enseñanza e investigación en psicología. México: Nueva Época. CNEIP.

Andrade, M., Miranda, C. \& Freixas, I., Rendimiento Académico y variables modificables en alumnos de 2do Medio de Liceos Municipales de la Comuna de Santiago Extraído el 18 de noviembre de 2005 desde:

http://www.unesco.cl/medios/biblioteca/documentos/eaprendizajes_rendimiento_academi co_2_medio_santiago.pdf?menu=/esp/atematica/evalcontexinterc/docdig.

Martínez, V. (1996). Factores determinantes del Rendimiento Académico en la enseñanza media. Psicología Educativa, Vol. II, 79,90.

Herrera, P. (1997) La Familia Funcional y Disfuncional, un indicador de salud. Rev. Cubana Med gen Integr; 13(6): 591-5. Extraído el 20 de Enero de 2007 desde:http://www.bvs.sld.cu/revistas/ped/vol71 199/ped06199.html.

Seguel, A., Holmes, Z., Venegas, K., Alcérreca, F., y Salamanca, R.(2000) Factores Socio Emocional Afectivos Y Separación De Los Padres En Niños De Tres A Cuatro Años. Extraído el 15 de noviembre de 2005 desde:

http://www.geocities.com/Heartland/Farm/8810/investig/separacl.html.

Adell, M (2002). Estrategias para mejorar el Rendimiento Académico de los Adolescentes. Ediciones Pirámide. 19-40.

Lamborn, S.D., Mounts N.S., Steinberg, L. \& Dornbush, S.M. (1991), Patterns of competence and adjustment among adolescents from authoritative, authoritarian, indulgent and neglectful families. Child development, 62, 5, 1049-1065.

Xinyin Chen (1997). Authoritative and Authoritarian Parenting Practices and Social and School Performance in Chinese Children. International Journal of Behavioral Development, $21(4), 855-873$. 
Londoño, V. (2005), Relación entre el estilo de autoridad ejercido por los padres de familia y el Rendimiento Académico de los niños y niñas entre los 8 y 11 años de edad escolarizados en los niveles 2 a 5 de básica primaria del municipio de ltaguí. Memoria para optar al título de psicóloga, Facultad de Psicología, Universidad de San Buenaventura, Medellín.

Casanova, P., García, C., De la torre, M., De la Villa, M., (2005), Influence of Family and SocioDemographic Variables on Students with Low Academia Achievement. Educational Psychology, 25(4), 423-435.

Lozano, A., (2003) Factores Personales, Familiares y Académicos que Afectan al Fracaso Escolar en la Educación Secundaria. Revista Electrónica de Investigación Psicoeducativa y Psicopedagógica, 1(1), 43-66.

Steinberg, Dornbusch \& Brown, (1992), Ethnic Differences in Adolescent Achievement: An Ecological Perspective. American Psychologist, 47(6), 723-729.

Covadonga, R.M., (2001), Factores Vinculados al Bajo Rendimiento Académico. Revista Complutense de Educación, 12(1), 81-113.

Espinoza, E., (2006), Impacto del Maltrato en el Rendimiento Académico. Revista Electrónica de Investigación Psicoeducativa, 4(9), 221 -238.

Gracia, Lila, Musitu, (2005), Rechazo Parental y Ajuste Psicológico y Social de los Hijos. Salud Mental, 28(2), 73-81.

Gracia, E., (2002), El maltrato infantil en el contexto de la conducta parental: Percepciones de padres e hijos. Psicothema, 14(2), 274-279.

Pérez, F., Fuster, E. \& Musitu O., (1988) Diferencias en los Tópicos de Comunicación entre Padres e Hijos según la Dirección de la Comunicación y las Variables Sexo, Edad y Status. Cuadernos de Consulta Psicológica, 4, 31 -41.

Quintana, J. (1993). Pedagogía Familiar. Madrid: Narcea.

Coll, C., Miras, M., Ornibia, J. \& Solé, I. (1998). Psicología de la Educación. Barcelona: Edhasa. 
Moreno, M.C., Cubero, R., (1990) Relaciones Sociales: Familia, Escuela, Compañeros, Años preescolares. En Marchesi, A., Coll, C., Palacios, J. (Eds). Desarrollo Psicológico y Educación. Madrid: Alianza.

Sorribes, M., García, F., (1996), Los Estilos Disciplinarios Paternos. En Clemente, R., Hernández, C. Contextos de Desarrollo Psicológico y Educación. (151-169), Aljibe.

Musito, G., Román, J., Gracia, E., (1988), Familia y educación: Prácticas educativas de los padres y socialización de los hijos. Barcelona: Labor.

Fuentes, M., Motrico, E., y Bersabé, R. (2001). Análisis Psicométrico de dos Escalas para Evaluar Estilos Parentales. Revista Psicothema, 13(4), 678-684.

Baumrind, D. (1968), Authoritarian v. Authoritative parental control. Adolescence, 3, 255-272.

Bronfenbrenner, U., (1987), La Ecología del Desarrollo Humano, Barcelona: Paidos.

Hernández, C., (1996), Bronfenbrenner y los Modelos Ecológicos. En R. Clemente, C. Hernández, Contextos de Desarrollo Psicológico y Educación; (91-110). Aljibe. 